\title{
DYNAMIC-FUZZY SIMULATION MODEL FOR REPRODUCTION OF MORTALITY CURVES
}

\author{
Andréia A. S. Schwaab \\ Dept. of Computing and Statistics \\ Federal University at Santa Catarina \\ Caixa Postal $476-88.040-900-$ \\ Florianópolis/SC - Brazil
}

Paulo J. de Freitas Filho

\author{
Dept. of Computing and Statistics \\ Federal University at Santa Catarina \\ Caixa Postal $476-88.040-900-$ \\ Florianópolis/SC - Brazil
}

\begin{abstract}
This paper presents the study and expansion of a dynamic simulation model for aging and death (Hargrove 1998), which contemplates the representation of physiological capacity and the generation of events of risk in the life of an individual. The study identified the most influential parameters in the results of the simulations and a health impact and recovery module was included. The simulation model incorporates a fuzzy module to treat uncertainty. The expansion conducted allowed adapting the results of the simulation to real mortality curves. The reproduction of mortality curves allowed the study of populations with similar characteristics as well as the factors that could influence their development. This is interesting principally because it is possible to calibrate parameters with risk values for diseases that have high associated costs for both public and private health plans.
\end{abstract}

\section{INTRODUCTION}

The system modeled is characterized by its complexity. The greatest difficulties are related to the events and their effects over time. For this reason, this project opted to use the precepts of continuous dynamic modeling. According to Thesen and Travis (1989), the finality of Continuous Dynamic Modeling is to capture gradual changes that occur over time caused by the controls, such as the gradual change that occurs when turning the steering wheel of a car. Studies in the health field with dynamic modeling have been conducted for applications such as: the dynamic of canine rabies (Ortega, Sallum and Massad 2000), the dynamism of HIV (Jafelice 2003), the functioning of the central nervous system (Nebot et al. 2003), smoking (Ahmad 2005; Ahmad and Franz 2008), aging (Penna 1995), etc.

In this work, the principal process modeled is that of aging. Various theories are used to explain aging, and can be classified in two large groups: (a) those based on progreammed events and (b) those based on random events. Nevertheless, even though they explore various factors involved in this process, these theories are not considered either totally valid or invalid (Hayflick 1997).

One consolidated equation is that described by the Gompertz' Theory (Gompertz 1825), which predicts that a mortality rate grows exponentially after 30 years of age. In equation (1), which is Gompertz' equation, $R_{m}$ represents the rate of mortality, $R_{0}$ the initial rate of mortality, $\alpha$ the rate of aging and $t$ the time.

$$
R_{m}=R_{0} e^{\alpha t}
$$

Over the years, it was realized that there was a need to readapt this idea to represent accidental risks to life. Thus the term $A$ of equation (2) comes to exercise this role.

$$
R_{m}=R_{0} e^{\alpha t}+A
$$

According to Satuffer (2007), while there are many theories that seek to explain aging, there are not many computer simulation models that seek to do so. Computer simulation models developed for this purpose enables studies regarding the factors associated to life evolution of the population. A widely known computational simulation model is the Penna model (1995), based on the theory of mutations (one of the theories of programmed events). Also based on the theory of mutations are the works of Stauffer and Radomski (2001), Maksymowicz et al. (1999), etc. 


\section{Schwaab and Freitas Filho}

The support model for the development of this study is that proposed by Hargrove (1998), created to replicate the behavior of an individual, from birth until death. For this reason the author delineated the physiological capacity of an individual and the generation of random events, responsible for the occurrence of diseases and accidents. The complementary work proposed here expand his model, allowing the inclusion of improvements regarding the treatment of uncertainty and better match the reality with the inclusion of a module of health impact and recovery. The model expanded in this study can be classified as belonging to the theories based on random events because the determination of the occurrence and intensity of the risk are random.

The preliminary results are encouraging. Once calibrated, the model allows replicating the behavior of a given population being studied and establishing parameters for it, to simulate the influence of specific variables. In the case of this research, the objective of the final model is to reproduce real curves of mortality, that is, given the frequency distribution of total deaths by age of a real population, is expected to achieve similar results by running the model. The following steps were investigated:

- Changes and expansion in the structure of the base model by using a fuzzy system to determine of the speed of recovery of the individuals considering their situations of risk.

- Identification of the principal parameters and their influence on the behavior of the model.

- Simulation of the temporal changes over time of morbimortality of groups of individuals.

\section{METHODOLOGY}

The methodology used was that of Systems Dynamics (Forrester 1961), allowing development of a continuous dynamic simulation model. This type of modeling allows better understanding of systems considered complex, which involve the relationship of a large number of variables over time (Kettenis 1982). The main diagrams which give support to this methodology are the stock-flow diagram and the causal-loop diagram (Forrester, 1961). The Flow Chart is the graphic representation of the simulation model and the principal tool for its development. In it, building blocks provide an understanding of the state variables (by means of stocks), of the transformations that took place in the variables (by means of flows) and of auxiliary elements, known as converters. In this work we use the Stella simulation environment (Stella isee Systems) to construct the Flow Chart. The causal-loop diagram shows the causal relationship between the elements of a system providing the identification of the type of relation (positive or negative) and the formation of feedback loops. On a positive causal relationship the changes in an element causes the same type of variation in the consequent element, for example, an increase in an element takes to an increase in the consequent element. In the negative case, if there is a decrease of one, the resulting factor will increase.

Hargrove's work, the suport model of this study, does not include a detailed study of the model and of the influence of the parameters on the final result. For this reason, more detailed studies of the basic model are justified as well as a proposal to expand that work in order to extend its applications. To understand and identify the parameters that most influence the behavior of the model, a preliminary investigative study was conducted with a complete factorial experiment design $\left(2^{k}\right)$. The parameters investigated were: growth rate, reserve rate, senescence rate, age independent risks, age dependent factors and risk factor. A total of sixty-four experiments were conducted (26 combinations). Each combination was replicated 1,000 times, representing the behavior of a population of one thousand individuals.

The preparation of the fuzzy logic model (Zadeh 1965) follows the steps of a fuzzy inference system in which the inputs are the data of the problem that should be addressed. These inputs undergo a process of fuzzyfication, which is a mapping of the membership function of the fuzzy sets defined. After the fuzzyfication, the amounts pass through the inference machine, which activates all the rules in parallel. Each rule generates a fuzzy set as an output, and at the end, all the outputs are added to form the resulting fuzzy set. Finally, the resulting set passes through defuzzification and become a numeric value.

\section{THE BASE MODEL}

The Hargrove model (Hargrove 1998), represented in Figure 1, offers a simulation of events that can occur during the life of an individual. The generation of events is conditioned on characteristics intrinsic to the individual and his or her environment. In addition, both the generation and the intensity of a risk event are of a stochastic nature. According to Hargrove (1998), the model incorporates stochastic characteristics because it is not known exactly when a risk situation will occur as well as the state of health of the individual, at that time, to confront the risk.

The structure of Figure 1 is composed mostly of converters that conduct the calculations that determine the future of an individual at each instant of time $(d t)$. Each simulation produces events for an individual, given that $n$ replications of a simulation represent the behavior of $n$ individuals with similar characteristics. 


\section{Schwaab and Freitas Filho}

The model considers that the characteristics of a person, with normal aging, are dependent on three parameters: growth rate, reserve rate, and senescence rate. These three factors define the Reserve, Aging and Function functions, which are responsible for the representation of some aspects of functional capacity. The Function element can be understood as a quantum of energy that everyone has until death. The Reserve is related to the functional capacity that increases until the adult phase and gradually decreases until the end of life. Aging is opposed to the Function and to Reserve, being a vector that causes the total capacity to decrease. The intensity of each element is calibrated by means of the parameters.

The model also contemplates the occurrence of accidental problems during a life (through the Age Independent Risk parameter) as well as the number of problems that can occur with age (through the Age Dependent Factors parameter, such as cardiovascular diseases). Based on these two parameters and on the Relative Capacity (resulting from the Total Capacity) of the subject, the occurrence of a risk event is determined, by the Monte Carlo stochastic method, which is executed at each step of the simulation. With this occurrence, the Risk Level will determine the intensity of the event. This value will be compared to a Survival Limit. If this limit is higher than the intensity of the event, the subject will survive the episode.

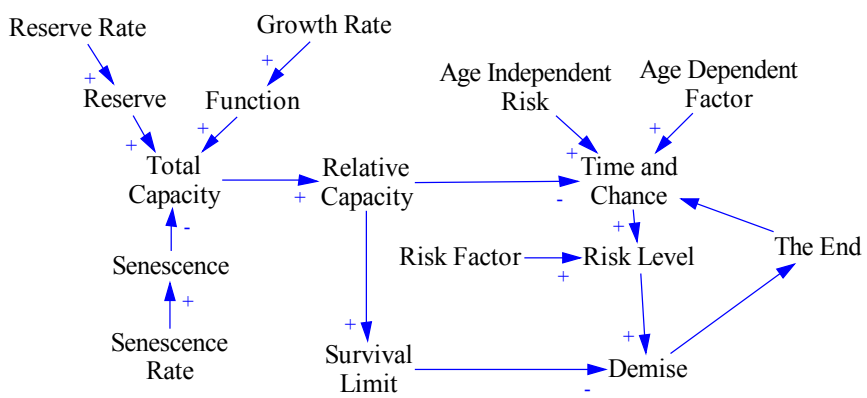

Figure 1: Structure of the Basic Model. Adapted from Hargrove (1998)

Figure 2 indicates that the function grows until the adult phase (approximately 25 years of age) and after this the value remains constant, given that this behavior corresponds to the activities of daily life responsible for our autonomy.

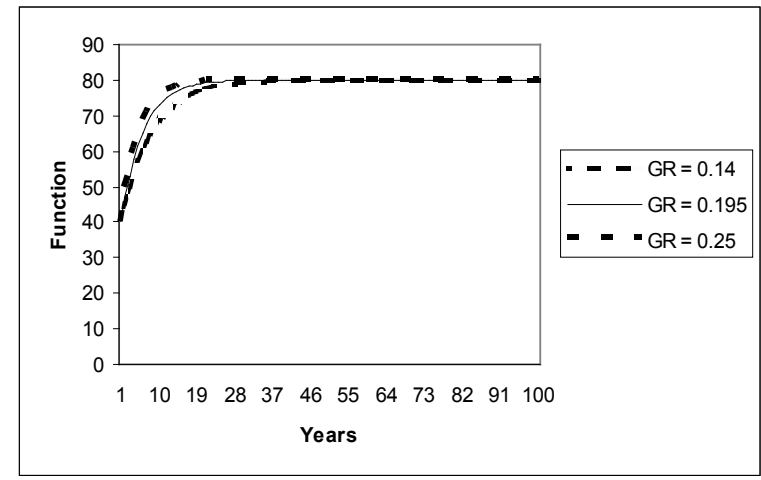

Figure 2: Behavior of the Function of an individual during a lifespan considering three different growth rates

Meanwhile, the Reserve (Figure 3) increases until approximately 15 years of age, and then decreases until the end of life. It represents the extra capacity that each organ has to resist greater than normal stresses (accidents, infections). 


\section{Schwaab and Freitas Filho}

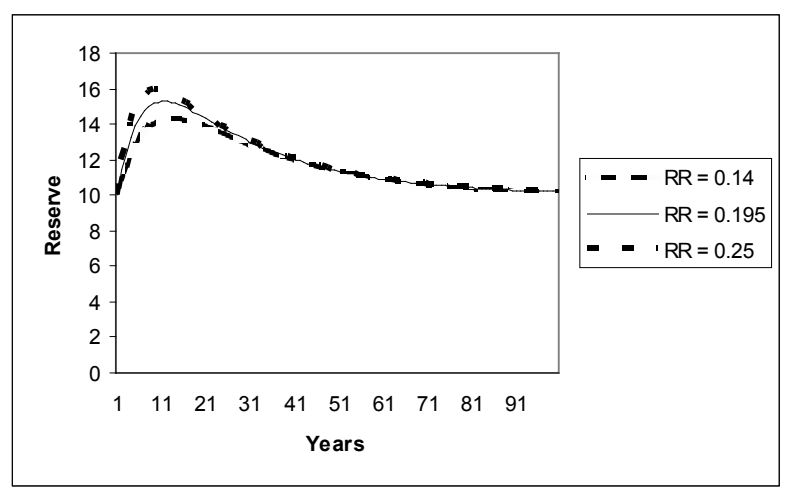

Figure 3: Behavior of the Reserve of an individual during his or her lifespan considering three different reserve rates

Aging begins at 25 years and increases over time. Although this seems precocious, it is a reality in terms of performance and is caused by cardiovascular aging (Figure 4).

The controllable factors are determined in the following manner:

- Growth Rate: specifies the speed of an individual's growth function. After finding the maximum value, the function remains constant for the rest of an individual's life. Considering $F$ the Function, $G R$ the Growth Rate and $T$ the time of simulation, the Function is calculated with equation (2).

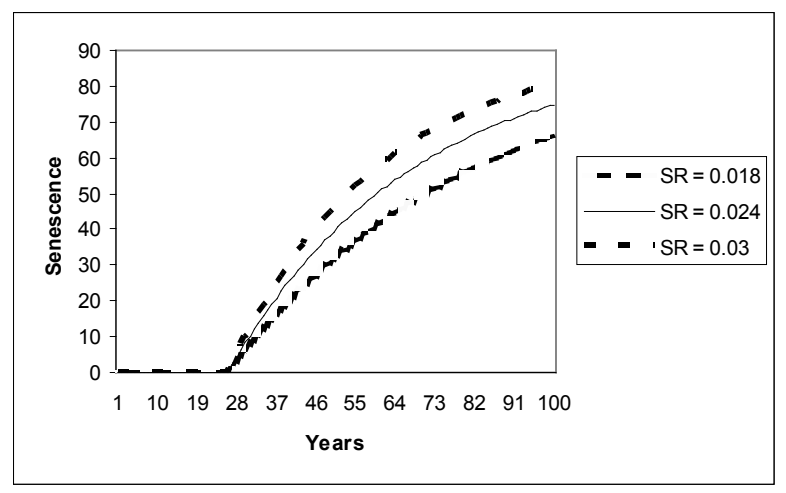

Figure 4: Behavior of the Aging of an individual considering three different senescence rates

$$
F=40+(40 \times(1-\exp (-G R \times T)))
$$

In equation (1) it can be seen that the minimum value of the Function is forty. With the increase of time, the result of the calculation of the exponential approximates to zero, which, at the maximum, causes the amount of $F$ to approximate to 80 . In Figure 2, it is possible to note that the greater the Growth Rate, the faster the maximum point is found.

- Reserve Rate: determines the speed of growth of the Reserve Function. With $R$ the Reserve and $R R$ the reserve rate, the function between these terms and the time is demonstrated by equation (3).

$$
R=10+10 \times(1-\exp (-R R \times T))-10 \times(1-\exp (-0.04 \times T))
$$

Figure 3 presents the reserve function from three different reserve rates. The higher the rate, the faster the growth of the function. Note that this function both grows and declines. The decline begins at the beginning of the adult phase.

- Senescence Rate: influences the Aging function. Senescence is a process that occurs after an individual's organism has reached a maximum stage of development, at around 25 years of age. The higher the Senescence Rate, the faster 


\section{Schwaab and Freitas Filho}

the individual ages. This function is expressed by equation (4). Consider S the Senescence and $S R$ the Senescence Rate.

$$
S=\left\{\begin{array}{l}
90 \times(1-\exp (-S R \times(T-25))), \text { se } T \geq 25 \\
0, \text { se } T<25
\end{array}\right.
$$

Figure 4 shows the application of three different senescence rates in equation (4). Note, both equation (4) and figure 4 indicate that aging begins at twenty five years of age and is more accentuated for higher rates.

- Age Independent Risk: is a value that represents any other type of risk that an individual may suffer, which is not related to his or her state of health, such as, the risk of accidents.

- Age Dependent Factor: represents the vulnerability to unexpected risks that can take place with age, such as, for example, exposure to pathogenic microorganisms, whose impact is greater in older people (Hargrove 1998).

- Risk Factor: aggregate values of Geller-Gesner survival tables (Hall and Zwemer 1979) which are calculated according to the health conditions of the public being studied. The calculation is thus realized in accordance, for example, with the presence of diseases (diabetes, high cholesterol, etc) and living habits (exercise, sedentariness, etc).

The Total Capacity is a characteristic determined by Function, by Reserve and by Aging. The Function and the Reserve add value to the Total Capacity. Senescence, in turn, causes the capacity of an individual to decrease. This can be observed by equation (5), where $T C$ is the Total Capacity, $F$ is the Function, $R$ is the Reserve, $S$ Senescence and $T$ time.

$$
T C=F+R-S-(0.1 \times T)-10 \times(1-\exp (-0.01 \times T))
$$

In equation (5), in addition to Senescence, there are other adjustments that reduce Total Capacity. These adjustments are based on the individual's age.

The Relative Capacity, based on the Total Capacity, is responsible for representing the effective capacity with which an individual will confront situations of risk, such as immunity, for example. In equation (6), consider $R C$ the Relative Capacity and $T C$ the Total Capacity.

$$
R C=\frac{T C}{87.67} \times 100
$$

The Survival Limit represents the existing force at a given moment to support life in a situation of risk. It means saying that if the occurrence of a risk had a value greater than the Survival Limit, the individual would not survive. In equation (7) note that the Survival Limit represents $45 \%$ of the total of the relative capacity. Consider $S L$ to be the survival limit and $R C$ the Relative Capacity.

$$
S L=0.45 \times R C
$$

The influence of three factors is necessary for an event that is a risk to health to take place: the relative capacity of the individual, the age independent risks and the age dependent factors. This can be observed in equation (8), which executes a Monte Carlos method. Consider TAC (Time and Chance) the decision of the occurrence of a Risk event, AIR the Age Independent Risks and $A D F$ the Age Dependent Factors.

$$
T A C=\text { MonteCarlo }\left(\frac{(2 \times A I R)+(A D F \times T)}{R C}\right)
$$

Equation (8) indicates that the weight of the age dependent factors increases with the years. Meanwhile, the age

independent risk is constant throughout a lifetime. Since the relative capacity decreases with time, the chances of events of risk increase. Figure 5 presents the relative capacity of an individual who has a Growth Rate $(G R)$ and Reserve Rate $(R R)$ equal to 0.14 ; and Senescence Rate equal to 0.018 . 


\section{Schwaab and Freitas Filho}

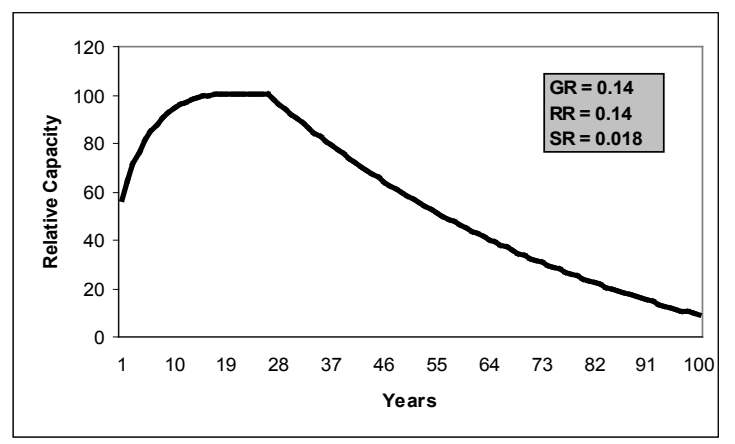

Figure 5: Relative Capacity of an individual

The Risk Level is the value resulting from the perspective of how much the occurrence of a risk event will mean for a person's health. Its significance will depend on the relative capacity and on the risk factor. Death occurs if the risk level is greater than the survival limit. The risk level is determined with equation (9). Consider $R L$ the risk, $R F$ the risk factor and $R C$ the relative capacity. The risk level is calculated only if the occurrence of a risk event is programmed. If there is an occurrence, the value of the level will be random.

$$
R L=\left\{\begin{array}{l}
\operatorname{Random}(0, R F \times R C), \quad \text { if } \quad T A C=1 \\
0, \quad \text { if } \quad T A C=0
\end{array}\right.
$$

\section{THE DESIGN OF THE EXPERIMENTS}

Table 1 shows the influence that each one of the factors causes in the average life time and in the mortality rate of the population until 100 years of age. It is possible to observe that at the lower level of the $A D F$ (Age Dependent Factors), the number of people who die before the age of 100 is reduced and the average life time is greater. Meanwhile, in the lower level of AIR (Age Independent Risk) the average life time increases and deaths decrease. In the upper level of the GR the life expectancy increases, but the number of total deaths nearly does not change. In the case of the RR, there were no significant changes for both the average life time and the average mortality, for the levels tested.

Table 1: The influence of factors in the mortality of a population.

\begin{tabular}{|c|c|c|c|}
\hline Factors & $\begin{array}{l}\text { Variations in } \\
\text { the levels }\end{array}$ & $\begin{array}{c}\text { Avg. } \\
\text { Lifetime }\end{array}$ & $\begin{array}{l}\text { Mortality } \\
\text { rate* }\end{array}$ \\
\hline \multirow{2}{*}{ Age Independent Risk } & + & $\downarrow$ & $\uparrow$ \\
\hline & - & $\uparrow$ & $\downarrow$ \\
\hline \multirow{2}{*}{$\begin{array}{c}\text { Age Dependent } \\
\text { Factors }\end{array}$} & + & $\downarrow$ & $\uparrow$ \\
\hline & - & $\uparrow$ & $\downarrow$ \\
\hline \multirow{2}{*}{ Growth Rate } & + & $\uparrow$ & --- \\
\hline & - & $\downarrow$ & --- \\
\hline \multirow{2}{*}{ Reserve Rate } & + & ---- & ---- \\
\hline & - & ---- & ---- \\
\hline \multirow{2}{*}{ Risk Factor } & + & $\downarrow$ & $\uparrow$ \\
\hline & - & $\uparrow$ & $\downarrow$ \\
\hline \multirow{2}{*}{ Senescence Rate } & + & $\downarrow$ & $\uparrow$ \\
\hline & - & $\uparrow$ & $\downarrow$ \\
\hline$\downarrow$ Decreases & \multicolumn{2}{|c|}{$\uparrow \quad$ Increases } & $+=$ Upper \\
\hline $\begin{array}{c}----=\text { There are no well- } \\
\text { defined trends for the values } \\
\text { observed }\end{array}$ & \multicolumn{2}{|c|}{$\begin{array}{c}* \text { Average rate observed up to } \\
100 \text { years of age }\end{array}$} & - = Lower \\
\hline
\end{tabular}




\section{Schwaab and Freitas Filho}

The experiments conducted indicate that the model was more sensitive to the parameters: Age Independent Risk, Risk Factor and Senescence Rate. In addition, the first two have a considerable interaction. The Reserve Rate, in turn, was the least significant factor in the results.

\section{THE PROPOSAL FOR EXPANSION OF THE MODEL}

The study conducted allowed identifying some issues that can be improved. The identification of these considerations was conducted with the assistance of specialists in the field.

The risk factor initially acted with the same intensity during a person's entire life. This is to say that upon birth, a person already has a risk factor equal to that it would be at the end of his or her life. With the change implemented, this amount acts in a distinct form for different age ranges, being more intense after the moment in which it is known that the person has a certain health problem. This modification was principally implemented by the fact that the risk factor represented a damage that is formed, above all, by living habits. This fact thus allows this amount to increase or decrease over time.

Another question considered is that in the occurrence of a risk event, the total capacity of an individual does not suffer. That is, if a individual suffers an accident to his or her health there is no depreciation for this event. This should not be the case, because given that it could lead to death, a risk event could generate a consequence. To change this condition, a new module was implemented that calculates the impact of the risk event, but that also allows recovery from a health problem. In this way, the model abbreviates the life at the time when a risk situation occurs and allows for recovery, observing the chances for survival and the current capacity for dealing with a risk situation.

The impact suffered can have a variety of interpretations such as consequences left by the risk event, which may be reduced over time, physical conditions that are momentarily affected, or even non-apparent conditions. Therefore, there are various types of interpretation of an impact. In this simulation model, the impact does not necessarily result in the deterioration of the physical condition or apparent physical state of the individual, but in how much his or her survival limit was affected.

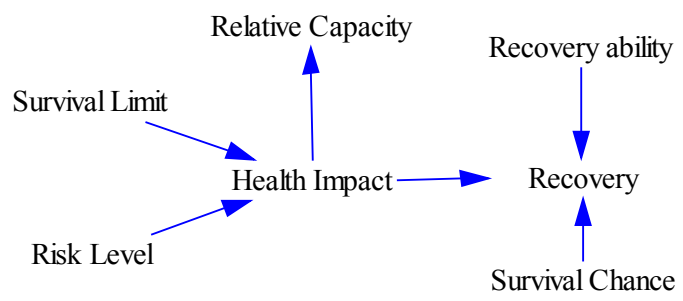

Figure 6: Module for impact and recovery

Figure 6 presents the module that calculates the impact and the recovery from a health problem. The Health Impact $(H I)$ depends on the survival limit and on the risk level and is calculated with equation (10).

$$
H I=\left\{\begin{array}{l}
\frac{R L}{S L}, \quad \text { if } \quad(R L>0 \text { and } S L>0 \text { and } R L>S L) \\
0, \quad \text { unless }
\end{array}\right.
$$

Equation (10) indicates that the impact represents the affected magnitude of the survival limit. This amount influences the relative capacity, the estimate for which is altered until there is improvement in the individual's health.

The recovery module was prepared with the assistance of specialists in the healthcare field. Given that there are no exact values for this representation, it was decided to use a fuzzy system, considering the age of the individual and the size of the impact. The recovery ability depends mainly on the age of the individual, which leads us to conclude that the higher the age, the lower the recovery ability. This chance for recovery is mentioned by Hayflick (1997), who explains that the recovery from an event, such as a fall, is different in young people and the elderly, given that it is slower for the latter. Concerning recovery, Finkelstein (2006) clarifies that the quality of the reduction of the accumualted damage during a lifetime decreases with increased age. Based on these ideas, the fuzzy system represents the ability to improve, that is, it determines the speed with which improvement can occur. This amount is modeled through a base of rules and fuzzy reasoning. 


\section{Schwaab and Freitas Filho}

The difficulty in finding mathematical functions capable of describing the variables involved is raised by Ortega, Barros and Massad (2003), who explains that for this reason the majority of the models are described through the experience of a specialist in the field.

\subsection{Implementation of the Fuzzy Module}

Artificial Intelligence techniques represent a suitable form of representation of knowledge. Fuzzy logic applications allow conducting inference in domains of uncertainty that may be characterized by imprecision and vagueness. For this reason, this study uses fuzzy modeling to handle one of the points of uncertainty for a model of senescence, that is the recovery ability of an individual facing a situation of risk.

The fuzzy inference module is constructed to verify the individual's speed of recovery. Because there is no knowledge about the type of problem the individual suffered, a generic means of representation can be considered. It is known that an individual, after surviving a risk situation, undergoes a period of recuperation that can vary from slow to fast. Or there may be no recovery. Thus, to realize this type of inference a modeling was conducted with the knowledge of healthcare specialists.

It was considered that the recovery depends on how the health was affected and the individual's age, and is thus not equal for everyone. There are no known mathematical functions capable of precisely determining the time needed for recovery. It is known, however, that healthcare specialists, through practical knowledge, have the ability to make qualitative determinations of this recuperation. This being the case, fuzzy modeling was chosen, given that the problem is characterized by haziness and imprecision. The proposal is for a system with a rule base that employs approximate reasoning in the determination of the speed of recovery given the occurrence of an impact to health. This will allow determining if the individual would have a fast or slow recovery.

The fuzzy system is formed by two inputs, age and impact suffered, and by an output that is recovery. During the simulation, these values are dynamically altered and the recuperation is then determined. Thus, three membership functions are created, two for the inputs and one for an output, with each one of them composed of four sets.

The age is determined for an interval of 0-100 years and is composed by the sets: child, youth, adult and elderly. Each one of these sets was prepared as a function of trapezoidal pertinence. The function of trapezoidal membership was chosen for this representation because it is simple and represents ranges of values that are approximately known. Figure 7 shows the sets defined by age.

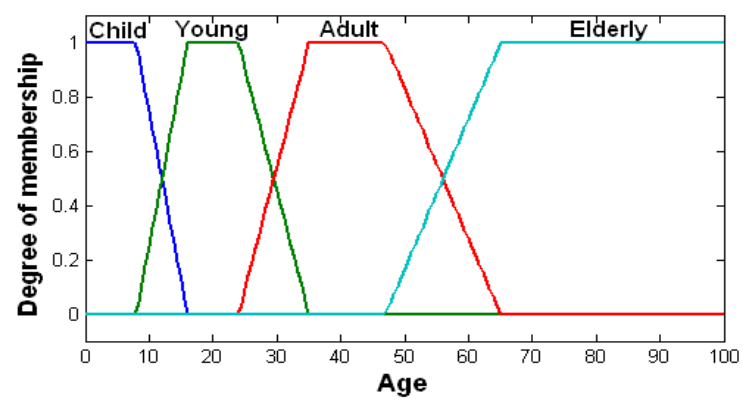

Figure 7: Membership function by age

To represent the impact, the membership function resulting from the product of the two sigmoid functions for the moderate and average sets was adopted; as well as the sigmoid for the weak and strong sets. This is because it involves an input with greater imprecision. It can be said that this is a case in which haziness predominates, or that is, there are no known values that allow reaching an approximation with a better defined range of values. The impact can be: weak, moderate, medium and strong. The impact can vary from $0-1$. The membership function for this input is presented in Figure 8 . 


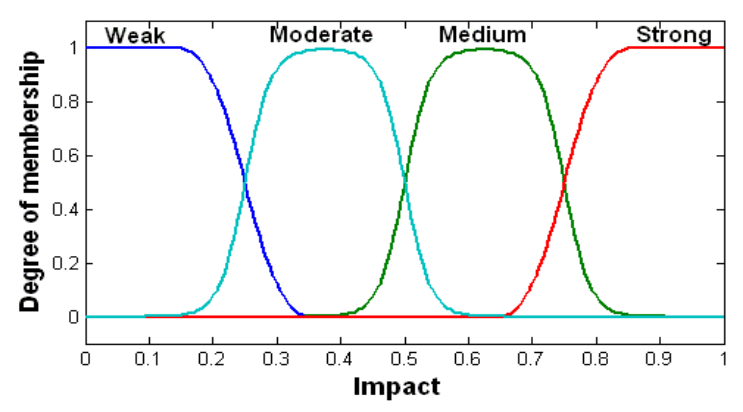

Figure 8: Membership function for the impact

To represent the recovery output the sigmoid function and the product of two sigmoid functions was also elected. This output can be classified as slow, moderate, medium or fast. The amounts that the system can assume vary from $0-1$. Figure 9 represents the definition of the sets of this output.

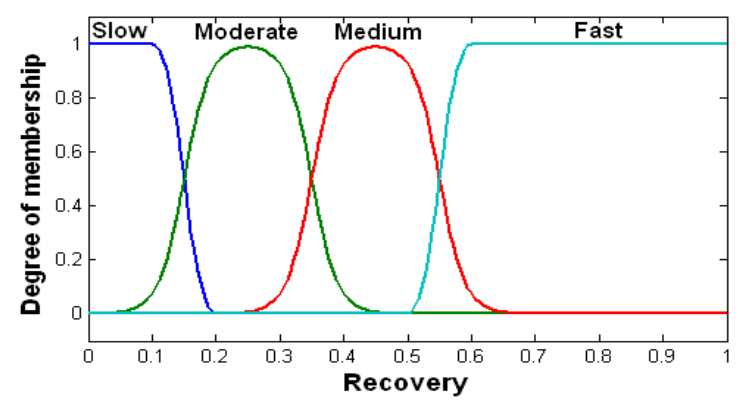

Figure 9: Membership function for the recovery from a heath problem

The construction of the rule base considered the fact that the vital function of the individual decreased with the passing of years, which, consequently caused the ability to recover to decrease, making the recovery process increasingly slower. The rule base used for the inference is presented in Table 2.

Table 2: Rule Base.

\begin{tabular}{l} 
1. If (age is child) and (impact is strong) then (recovery is moderate) \\
2. If (age is child) and (impact is medium) then (recovery is medium) \\
3. If (age is child) and (impact is moderate) then (recovery is fast) \\
4. If (age is child) and (impact is weak) then (recovery is fast) \\
5. If (age is youth) and (impact is strong) then (recovery is medium) \\
6. If (age is youth) and (impact is medium) then (recovery is medium) \\
7. If (age is youth) and (impact is moderate) then (recovery is fast) \\
8. If (age is youth) and (impact is weak) then (recovery is fast) \\
9. If (age is adult) and (impact is strong) then (recovery is moderate) \\
10.If (age is adult) and (impact is medium) then (recovery is moderate) \\
11.If (age is adult) and (impact is moderate) then (recovery is medium) \\
12.If (age is adult) and (impact is weak) then (recovery is medium) \\
13.If (age is elderly) and (impact is strong) then (recovery is slow) \\
14.If (age is elderly) and (impact is medium) then (recovery is slow) \\
15.If (age is elderly) and (impact is moderate) then (recovery is slow) \\
16.If (age is elderly) and (impact is weak) then (recovery is moderate) \\
\hline
\end{tabular}

The parameters of the fuzzy system were determined through simulations. A result was reached that is in keeping with the expectations (based on mortality data for real populations) using the following methods for inference: 
- And (t-norma): product

- Implication: product

- Aggregation: maximum

- Defuzzification: som (smallest of maximum)

The response surface is presented in Figure 10. It reveals that for high values of age and impact the recovery has low values. While for low input values, the output is higher.

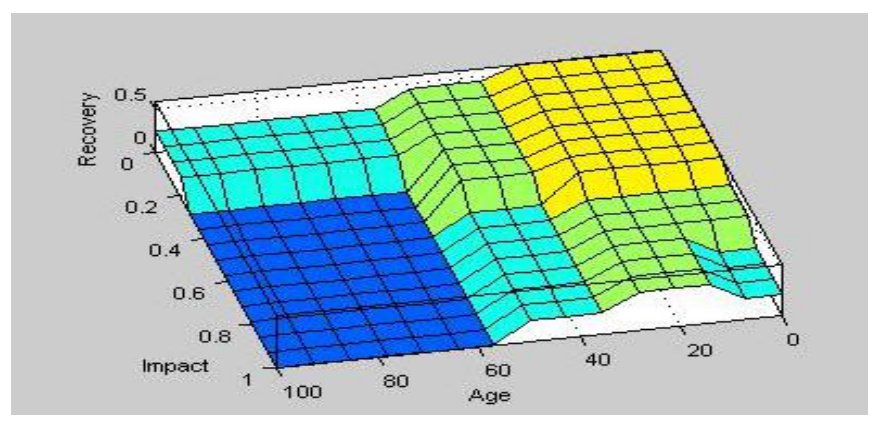

Figure 10: Response surface

\section{RESULTS}

To validate the results found by the model, adjustments to the parameters were realized so that it would be possible to reproduce the mortality curve for Santa Catarina State, Brazil. The information used was derived from a database of the federal government (DATASUS - Database of the Unified Health System). We took the overall mortality values for the year 2007, which a total of 31,106 people distributed by age classes. Since it concerns the mortality for the state as a whole, any one specific disease is not considered. Therefore, the risk factor, which is responsible for the representation of the health condition and the living habits, had its values associated to health conditions for which the risk of death is not very high. The results expected could be achieved with the following parameters:

- Growth Rate: 0.14

- Senescence Rate: 0.018

- Reserve Rate: 0.05

- $\quad$ Age Independent Risk: 100

- Age Dependent Factors: 4

The risk factor varied with the age ranges, beginning at 0.485 and terminating at 0.70 . Considering the age ranges in question, the significance of these values for the risk factor, indicates a state of health considered reasonable. This would be, for example, the risks attributed to people who are non-smokers or who are smokers in recovery (Hall and Zwemer 1979). That is, the fact that a person is 80 years old with a risk factor of 0.70 does not mean that he has grave problems.

The graph in Figure 11 indicates the curve generated by the model, the true curve and the curves of the confidence interval, considering $\alpha=0.01 \%$. 


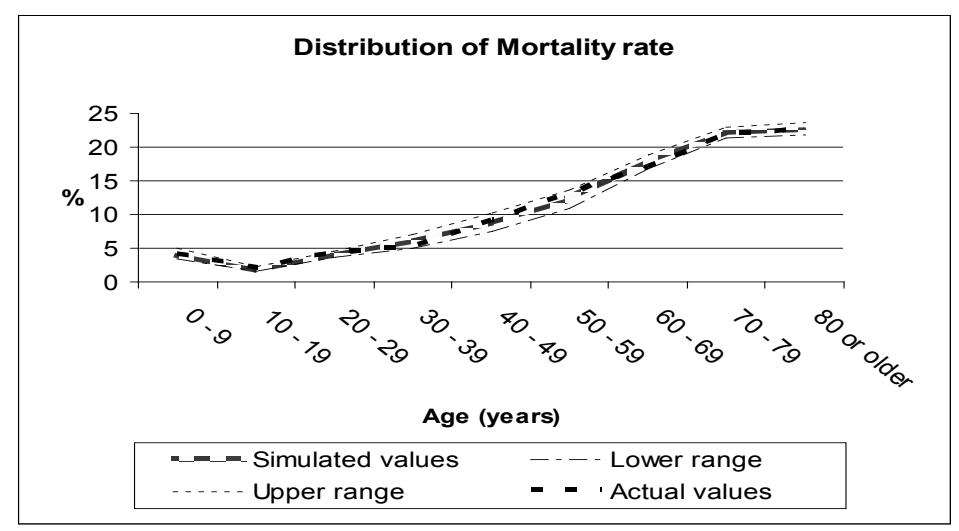

Figure 11: Results of the simulator with the implementation of changes in the model

Figure 11 allows observing that the result achieved by the model is in keeping with the reality observed. Figure 12 presents the results generated by the original model, which with the same values for the parameters, does not achieve a result close to reality.

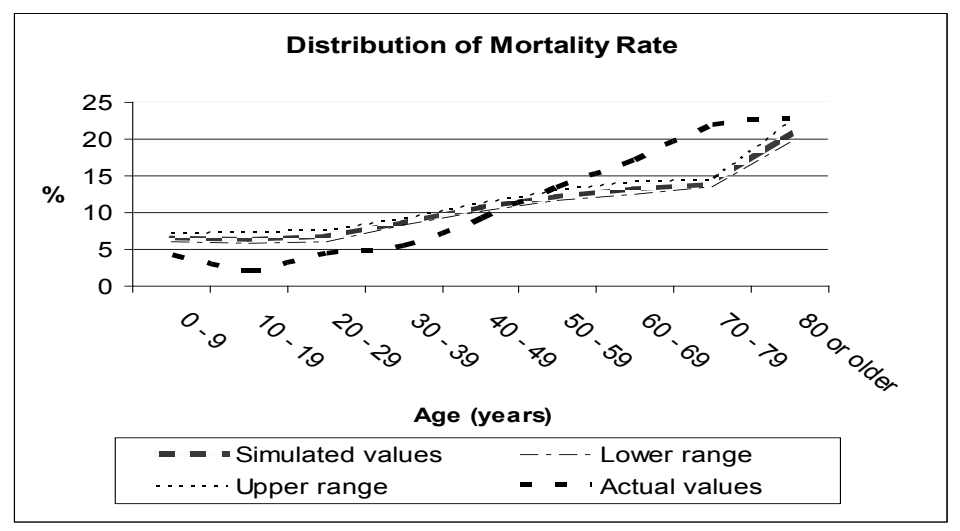

Figure 12: Results of the simulator of the original model

\section{CONCLUSIONS}

This work presents the study and expansion of a simulation model of aging and death. To do so, it began with the work of Hargrove (1998), which was important for the representation of physiological aging, but which did not suitably represent some situations that take place during and after the occurrence of risk events, such as, the decline of the ability to recover.

The experimental design showed that the Age Independent Risks, Risk Factor and Senescence Rate are those which most influence the simulation's results. This result collaborated in the determination of the values of the parameters, and indicated that the parameters not related to natural aging have notable influence on the results.

The changes implemented in the base model (the impact and the fuzzy recovery module) allowed smoother results. This is because the change in the values of the most influential parameters caused sharp changes in the results. Moreover, the amount set for the risk factor does not allow notable distinctions in mortality between the age groups. In this way, the impact and the fuzzy recovery module contributes to a situation of equilibrium. The simulation technique in conjunction with a specialist fuzzy system allows proper modeling of uncertainty caused by imprecision - which is highly present in the elements involved in aging. This type of modeling allows that the experience of specialists in the fields to be represented in order to contribute to the validation of the model through its imitation of reality.

The modeling of the fuzzy system proves to be suitable for this context, considering that there are no precise mathematical representations for the calculation of recovery of an individual. In general, the module contributes to the realization of distinctions between capacities for recovery, so that individuals who suffer impacts and have different ages can be particularized.

The results of the changes realized on the base model were validated for the mortality curve for Santa Catarina State Brazil. It was found that the expanded model made the results of the simulation more robust. The model can be of great 


\section{Schwaab and Freitas Filho}

importance, because it is sensitive to variables that are routinely collected in epidemiological data bases and survival tables for risk factors. In addition, it is now sensitive to modifiable factors that increase the morbimortality of a population. By allowing the replication of morbidity curves, it can be applied to simulate the impact of policies and interventions for the promotion of long term health of populations, as well as for the realization of calculations and planning of health care costs for example. To be applied to other realities, it is necessary to observe population development factors, such as economic and social issues, in the determination of parameters.

\section{REFERENCES}

Ahmad, S. 2005. Increasing excise taxes on cigarettes in California: a dynamic simulation of health and economic impacts. Preventive Medicine 41(1):276-283.

Ahmad, S., and G. A. Franz. 2008. Raising taxes to reduce smoking prevalence in the USA: A Simulation of the anticipated health and economic impacts. Public Health 122(1):3-10.

Finkelstein, M. S. 2006. On engineering reliability concepts and biological aging. MPIDR Working Papers WP-2006-021, Max Planck Institute for Demographic Research, Rostock, Germany.

Forrester, J. W. 1961. Industrial Dynamics. Cambridge, Massachusetts: MIT Press.

Gompertz, B. 1825. On the nature of the function expressive of the law of human mortality, and on a new mode of determining the value of life contingencies. Philosophical Transactions of the Royal Society 115:513-585.

Hall, J. H., and J. D. Zwemer. 1979. Prospective Medicine. Indianopolis, Indiana: Methodist Hospital of Indiana.

Hargrove, J. L. 1998. Dynamic Modeling in the Health Sciences. Springer-Verlag New York, Inc.

Hayflick, L. 1997. Como e por que envelhecemos. Rio de Janeiro: Campus.

Jafelice, R. M. 2003. Modelagem Fuzzy para Dinâmica de Transferência de Soropositivos para HIV em Doença Plenamente Manifesta. Tese de Doutorado, UNICAMP, Campinas, São Paulo, Brasil.

Kettenis, D. L. 1982. Is there any need for combined continuous discrete simulation languages?. SIGSIM Simul 13(1-4):1222.

Maksymowicz, A. Z., M. Bubak, K. Zajac, and M. Magdón. 1999. Computer Simulation of Ageing with an Extended Penna Model. In Proceedings of the 7th international Conference on High-Performance Computing and Networking, ed. P. M. Sloot, M. Bubak, A. G. Hoekstra, and B. Hertzberger, 43-49 . London: Springer-Verlag.

Nebot, A., F. Mugica, F. E. Cellier, and M. Vallverdú. 2003. Modeling and Simulation of the Central Nervous System Control with Generic Fuzzy Models. Simulation 79(11): 648-669.

Ortega, N. R. S., P. C. Sallum, and E. Massad. 2000. Fuzzy dynamical systems in epidemic modelling. Kybernetes 29(12):201-218.

Ortega, N. R. S., P. C. Sallum, and E. Massad. 2003. Fuzzy Gradual Rules in Epidemiology. Kybernetes 32(4):460-477.

Penna, T. J. P. 1995. A Bit String Model For Biological Aging, Journal of Statistical Physics 78:1629.

Stauffer, D., and J. P. Radomski. 2001. Social effects in simple computer model of aging. Experimental Gerontology 37(1): $175-180$.

Stauffer, D. 2007. The Penna Model of Biological Aging. Bioinformatics and Biology Insights 2007(1):91-100.

Stella, isee Systems.

Thesen, A., and L. E. Travis. 1989. Simulation for decision making: an introduction. In Proceedings of the 21st Conference on Winter Simulation, ed. E. A. MacNair, K. J. Musselman, and P. Heidelberger, 9-18. Washington, D.C., United States: ACM.

Zadeh, L. A. 1965. Fuzzy Sets. Information and Control 8:338-353.

\section{AUTHOR BIOGRAPHIES}

ANDRÉIA ALVES DOS SANTOS SCHWAAB. B.S. (2006) - Computer Science, State University of Maringá, UEM. Student in the Graduate Program in Computer Science (PPGCC) at the Federal University at Santa Catarina, UFSC. Experience in Computer Systems and Artificial Intelligence. Her e-mail is<alves.andreia@inf.ufsc.br>.

PAULO JOSÉ DE FREITAS FILHO. B.S. (1978) - Metallurgical Engeneering, Federal University at Rio Grande do Sul, UFRGS, Brazil. Master's (1984) - Production Engeneering, Federal University at Santa Catarina, UFSC, Brazil. Doctoral (1994) - Production Engeneering, UFSC. Associate Prof. At UFSC. Principal research interest in modeling and simulation, performance evaluation and systems capacity planning. His e-mail is $<$ freitas@inf.ufsc.br>. 